\title{
Population-based survey on prevalence of adult patients with epilepsy in Taiwan (Keelung community-based integrated screening no. 12)
}

\author{
Chih-Chuan Chen ${ }^{a}$, Ta-Fu Chen ${ }^{a}$, Yuarn-Chung Hwang ${ }^{b}$, Ying-Rong Wen ${ }^{c}$, \\ Yueh-Hsia Chiu ${ }^{\mathrm{d}}$, Chia-Yun Wu ${ }^{\mathrm{d}}$, Rong-Chi Chen ${ }^{\mathrm{a}, \mathrm{e}}$, \\ Tony Hsiu-Hsi Chen ${ }^{\mathrm{d}}$, Horng-Huei Liou ${ }^{\mathrm{a}, *}$ \\ a Department of Neurology and Pharmacology, National Taiwan University Hospital and National Taiwan University, \\ College of Medicine, 1, Sec. 1, Jen-Ai Road, Taipei 100, Taiwan \\ ${ }^{\mathrm{b}}$ Department of Neurology, Keelung Hospital, Department of Health, Executive Yuan, 268, Xin 2nd Road, \\ Keelung City 201, Taiwan \\ ${ }^{\mathrm{c}}$ Department of Neurology, Farestern Poly-Clinic, 8, Yung-Sui Street, Taipei 100, Taiwan \\ ${ }^{\mathrm{d}}$ Institute of Preventive Medicine, College of Public Health, National Taiwan University, 17, \\ Hsu-Chow Road, Taipei 100, Taiwan \\ e Department of Neurology, En Chu Kong Hospital, 399 Fuhsing Road, Sanhsia, Taipei 237, Taiwan
}

Received 28 April 2006; received in revised form 23 June 2006; accepted 7 July 2006 Available online 30 August 2006

\begin{abstract}
Purpose: To determine the prevalence rate and patterns of adult patients with epilepsy in Taiwan, we conducted a communitybased neuroepidemiological survey.

Methods: Epilepsy was detected by neurologists using one-stage method. It was integrated into a community health screening service and performed from 1 January 2001 to 31 December 2001 in Keelung, a northern city in Taiwan. A total of 13,663 subjects aged 30 years or older participated in this survey.

Results: There were 52 patients with epilepsy in this study. Among them, 37 were patients with active epilepsy. The age-adjusted prevalence rate of active epilepsy above 30 years old was $2.77 / 1000$ (to the 1980 US population) with the highest rate in subjects aged 40-49 years (4.0/1000). There was a trend of higher prevalence rate in male than in female. The most common seizure type was complex partial seizure (46.0\%). Using one-stage detection method, we found nine (24.3\%) patients with active epilepsy who had never been diagnosed before. Among the patients with active epilepsy, 35.1\% were symptomatic cases. Head injury (13.5\%) is the leading cause, followed by CNS infection $(8.1 \%)$, stroke $(5.4 \%)$ and perinatal insult $(5.4 \%)$. The lifetime prevalence rate of epilepsy (including active epilepsy and epilepsy in remission) was 3.14/1000 for age above 30 years.
\end{abstract}

\footnotetext{
* Corresponding author at: Department of Neurology and Pharmacology, College of Medicine, National Taiwan University Room 1141, No. 1, Sec. 1, Jan-Ai Road, Taipei 100, Taiwan. Tel.: +8862 23123456x8325; fax: +886223915297.

E-mail address: hhliou@ha.mc.ntu.edu.tw (H.-H. Liou).
} 
Conclusions: Comparing to previous epilepsy survey in 1993, our results showed that the prevalence rate of epilepsy was rather stable over the past decade in northern Taiwan. Head injury is the leading cause responsible for active epilepsy. Improving public safety is an important public health issue which may help to reduce occurrence of epilepsy.

(C) 2006 Elsevier B.V. All rights reserved.

Keywords: Seizure; Epilepsy; Prevalence; Keelung; Taiwan

\section{Introduction}

Epilepsy is a common neurological disorder. As there are a variety of underlying etiologies and investigation methods, epidemiological profiles vary widely across countries and regions with the reported prevalence rates mostly ranging from $4 / 1000$ to $10 / 1000$ (for review see ILAE, 1997; Forsgren et al., 2005). But extremely high prevalence rate (57/1000) had also been reported (Gracia et al., 1990). Most of these findings are based on cross-sectional surveys, and there were rare studies addressing whether prevalence rate has increased or decreased after a period in a country or region. In a longitudinal study, Hauser et al. (1991) reported an increasing prevalence rate from 1940 to 1980 in Rochester, Minnesota. Information on time series of prevalence rates was hardly available in one or equivalent community-based study. Comparison of two surveys at different times may be helpful to look at the trend regarding the change of prevalence rate and underlying etiology.

In 1993, there was one large-scaled two-stage doorto-door survey of epilepsy which revealed the crude prevalence rate of active epilepsy was 2.7/1000 in populations above 40 years old in Taiwan ( $\mathrm{Su}$ et al., 1998). The prevalence rate was lower than reports from most other countries. After approximately 10 years, we conducted another community-based epidemiological study of epilepsy in Keelung, the most northern city in Taiwan, by using one-stage detection method. The aim of this study is to determine the prevalence rate and etiologies of epilepsy in Taiwan and the trend across 10 years.

\section{Methods}

\subsection{Study areas and population}

We conducted a survey for epilepsy in Keelung, a northern city in Taiwan (Fig. 1). This survey was one part of a community screening service: Keelung community-based integrated screening (KCIS). The implementation and preliminary findings of KCIS are described in detail elsewhere (Chen et al., 2004; Tung et al., 2004; Hsu et al., 2005; Chiu et al., 2006). Briefly, the KCIS screening program is a multiple disease screening service which targeted at residents aged over 30 years old living in Keelung. This screening service was provided between 1 January 1999 and 31 December 2001. All seven administrative regions within this city were covered. Pap smear screening was the basis for integrating other screening regimens into KCIS study, including tests for breast, liver, colorectal and oral cancer, type II diabetes mellitus, hypertension, hyperlipidemia, and neurological disorder screening. From 1 January 2001 to 31 December 2001 the epilepsy survey was integrated into this screening service.

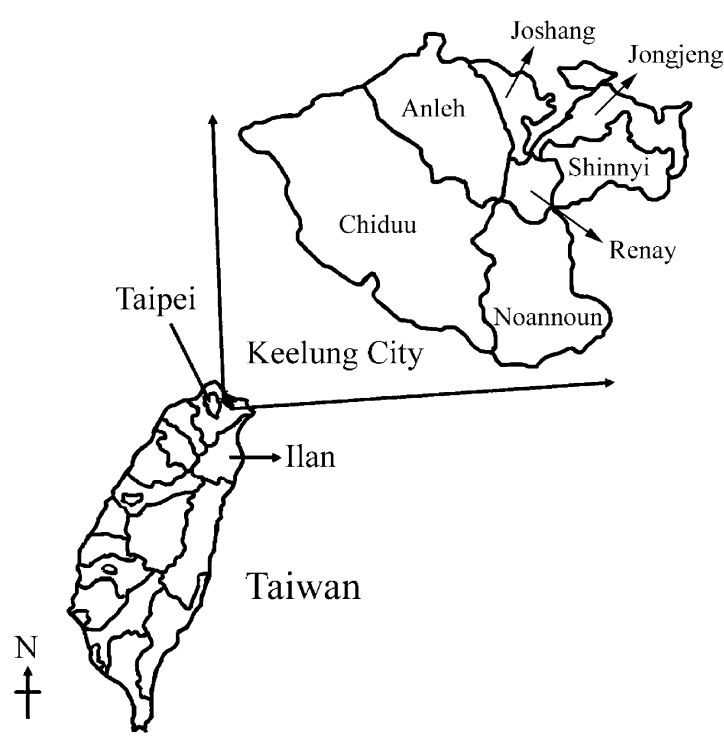

Fig. 1. Keelung city is the northernmost city of Taiwan. It is located near Taipei, the capital of Taiwan. It consists of seven administrative regions. The Keelung community-based integrated screening is survey covered all regions. Previous epidemiological study was performed at Ilan in 1993. Ilan is located in the northeast area of Taiwan. 


\subsection{Diagnosis procedures and definitions of symptomatic epilepsies}

In the place of screening service activity, the participating residents were given written informed consent. Each subject first answered one screening questionnaire. There were well-trained social workers to help people writing this questionnaire. The details of the questionnaire were shown in Appendix A. Then each subject was evaluated by board-certified neurologists form National Taiwan University Hospital (Drs. C.C. Chen, T.F. Chen, Y.C. Hwang, Y.R. Wen and H.H. Liou).

The overall validity of this instrument was measured in a hospital sample of 30 patients with epilepsy; and the sensitivity was $100 \%$. Specificity was investigated in 30 hospital visitors free of epilepsy and other diseases revealed $95 \%$. Each subject was evaluated by a neurologist to determine whether he or she is a patient with epilepsy on the basis of information on screening questionnaire, history taking and neurological examination.

In this study, acute symptomatic seizure is defined as acute provoked seizure occurring in the immediate context of an injury or event that impairs or alters brain function. Epilepsy is defined as two or more clinical recurrent unprovoked seizures not related to withdrawal of drugs or an acute metabolic event. Patient with active epilepsy is the individual had had seizures or continued receiving antiepileptic drugs within 5 years. The International League Against Epilepsy (ILAE, 1981) classification of seizure was used for diagnosis/classification of seizure types. Electroencephalography (EEG) and neuroimage study such as CT scan were not essential for diagnosis. But the uncertain cases would be referred to Keelung hospital for examinations to provide addition information. The final diagnosis and classification of seizure type and epilepsy etiology were discussed and determined in a consensus meeting by all neurologists participated in this study. All the patients with seizure and epilepsy were follow-up at Keelung hospital. The patients were classified as "old" or "new" cases. Old cases were those who had been previously diagnosed as epilepsy by other hospitals. New cases were patients with epilepsy that first detected by this survey. They were unrecognized their disease and have never been treated by any neurologist or traditional physicians.
The major causes of remote symptomatic epilepsy are defined according to guidelines for epidemiologic studies on epilepsy from ILAE commissions (ILAE, 1993).

\subsection{Statistical method}

Prevalence was calculated as the number of cases per 1000 population. The $95 \%$ confidence intervals were calculated by asymptomatic method. The pointdates were from 1 January 2001 to 31 December 2001. To compare with other reports, we calculated age-adjusted rate by using 1980 US population. Age and gender specific prevalence rates of active epilepsy were calculated. Lifetime prevalence rate of epilepsy was calculated by including both patients with active epilepsy and epilepsy in remission on the prevalence date (Hauser et al., 1991).

\section{Results}

\subsection{Prevalence of epilepsy}

The overall 16,767 participants aged 30 years or more of KCIS program were simultaneously invited to epilepsy screening. A total of 13,663 subjects (5014 male and 8649 female) were evaluated in this epilepsy survey by neurologists. The overall attendance rate of epilepsy screening was $81.5 \%$. There is no striking difference in subgroups of gender and age groups. Attendance rate of each age group and gender was more than $85 \%$ except for young age group (aged 30-39, 70.9\%). Among those participants, 52 were diagnosed as patients with epilepsy and 17 as acute symptomatic seizure. The 17 patients with acute symptomatic seizure were not considered as epilepsy because their seizures were related to acute provoked events. Thirty-seven cases out of 52 lifetime patients with epilepsy were active epilepsy. Table 1 shows the age- and gender-specific prevalence rates of adult patients with active epilepsy in this study. Data of previous study at Ilan, Taiwan was also provided for comparison. The crude prevalence rate of active epilepsy above 30 years old in Keelung was $2.7 / 1000$ (95\% CI: $1.85-3.58$ per 1000 ). Age-adjusted prevalence rate was 2.77/1000 (95\% CI: 2.21-3.33 per 1000). The prevalence rate was higher 

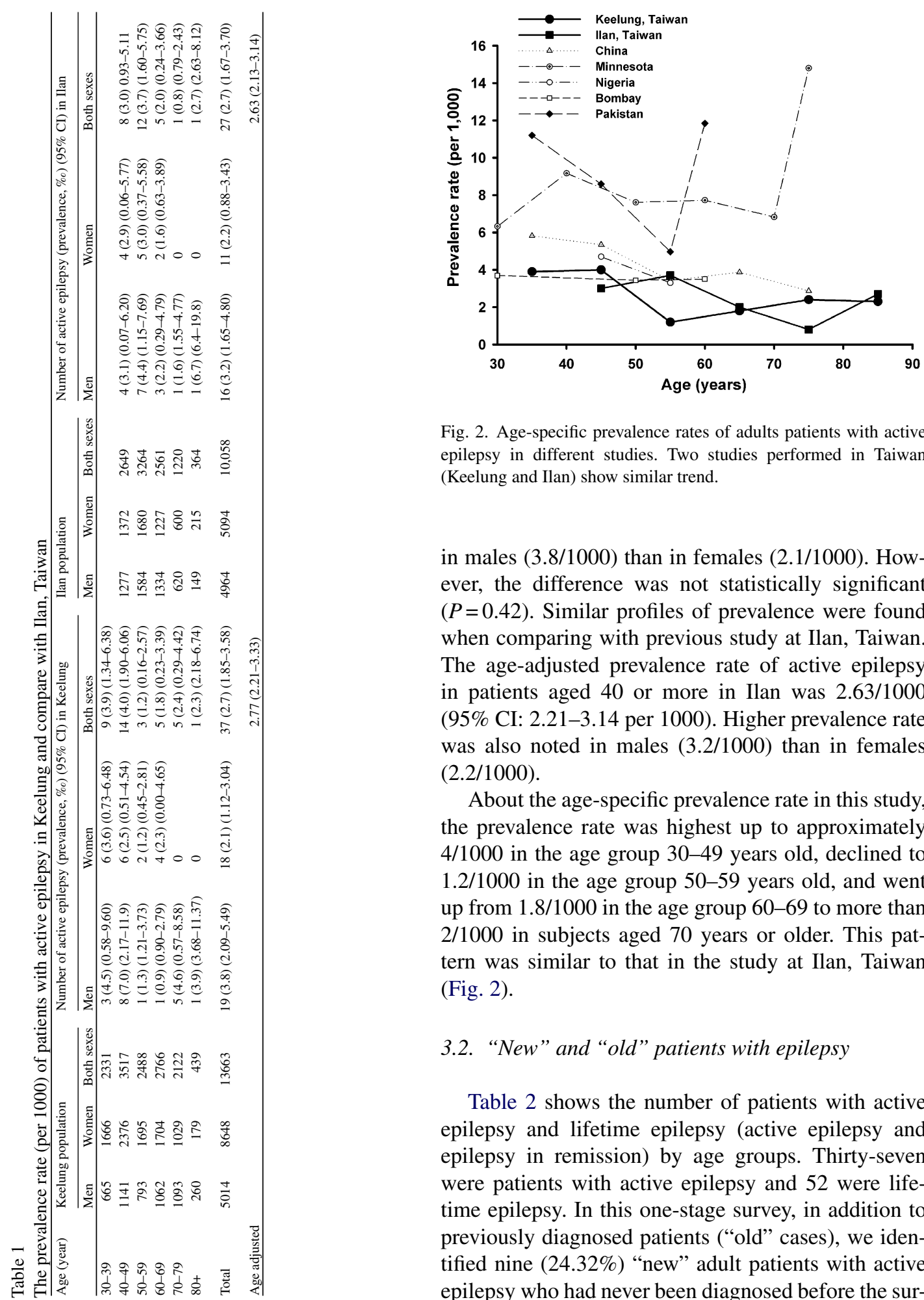

Fig. 2. Age-specific prevalence rates of adults patients with active epilepsy in different studies. Two studies performed in Taiwan (Keelung and Ilan) show similar trend.

in males $(3.8 / 1000)$ than in females $(2.1 / 1000)$. However, the difference was not statistically significant $(P=0.42)$. Similar profiles of prevalence were found when comparing with previous study at Ilan, Taiwan. The age-adjusted prevalence rate of active epilepsy in patients aged 40 or more in Ilan was $2.63 / 1000$ (95\% CI: $2.21-3.14$ per 1000). Higher prevalence rate was also noted in males (3.2/1000) than in females $(2.2 / 1000)$.

About the age-specific prevalence rate in this study, the prevalence rate was highest up to approximately $4 / 1000$ in the age group $30-49$ years old, declined to $1.2 / 1000$ in the age group 50-59 years old, and went up from $1.8 / 1000$ in the age group 60-69 to more than $2 / 1000$ in subjects aged 70 years or older. This pattern was similar to that in the study at Ilan, Taiwan (Fig. 2).

\section{2. "New" and "old" patients with epilepsy}

Table 2 shows the number of patients with active epilepsy and lifetime epilepsy (active epilepsy and epilepsy in remission) by age groups. Thirty-seven were patients with active epilepsy and 52 were lifetime epilepsy. In this one-stage survey, in addition to previously diagnosed patients ("old" cases), we identified nine (24.32\%) "new" adult patients with active epilepsy who had never been diagnosed before the sur- 
Table 2

Number of new (first diagnosed) and old (previously diagnosed) patients with epilepsy in this survey

\begin{tabular}{|c|c|c|c|c|c|c|c|}
\hline \multirow[t]{2}{*}{ Age } & \multicolumn{3}{|c|}{ Number of active epilepsy } & \multicolumn{4}{|c|}{ Number of lifetime epilepsy (prevalence, \%o) (95\% CI) } \\
\hline & New & Old & Total & New & Old & Total & Prevalence $(95 \% \mathrm{CI})$ \\
\hline $30-39$ & 3 & 6 & 9 & 5 & 8 & 13 & $5.58(2.55-8.60)$ \\
\hline $40-49$ & 2 & 12 & 14 & 5 & 16 & 21 & $5.97(3.42-8.52)$ \\
\hline $50-59$ & 0 & 3 & 3 & 1 & 4 & 5 & $2.01(0.25-3.77)$ \\
\hline $60-69$ & 2 & 3 & 5 & 2 & 4 & 6 & $1.81(0.22-3.39)$ \\
\hline $70-79$ & 2 & 3 & 5 & 2 & 4 & 6 & $2.36(0.29-4.42)$ \\
\hline $80^{+}$ & 0 & 1 & 1 & 0 & 1 & 1 & $2.28(-2.18-6.74)$ \\
\hline Total & 9 & 28 & 37 & 15 & 37 & 52 & $3.14(2.651-4.67)$ \\
\hline
\end{tabular}

Lifetime epilepsy included active epilepsy and epilepsy in remission.

vey. The lifetime crude prevalence rate of epilepsy was 3.14/1000 (95\% CI: $2.65-4.67$ per 1000) with up to $28.85 \%(15 / 52)$ were newly detected in this survey.

\subsection{Seizure types and etiologies of epilepsy}

More than half patients with active epilepsy have partial onset seizures. The most common type of seizure in patients with active epilepsy above 30 years old was complex partial seizure (46.0\%), followed by partial seizure with secondarily generalization (32.4\%) and generalized seizure (18.9\%).

Among the patients with active epilepsy, only $35.1 \%$ could be identified as remote symptomatic cases whereas $64.9 \%$ patients were idiopathic or cryptogenic. Table 3 shows the etiologies by age group. The leading cause of remote symptomatic cases was head injury (13.5\%), followed by CNS infection $(8.1 \%)$, stroke $(5.4 \%)$ and prenatal insult $(5.4 \%)$. All the five epilepsy patients caused by head injury were male and $80 \%$ of them (4/5) were at younger age groups (30-49 years old).

\section{Discussion}

\subsection{The prevalence rates of epilepsy across 10 years in Taiwan}

The present community-based epidemiological study using one-stage method revealed the adjusted prevalence rate of active epilepsy above 30 years old was 2.77/1000 in Keelung, Taiwan. Previous largescaled neuroepidemiological survey of epilepsy in Ilan, another county next to Keelung in Taiwan, with twophase door-to-door survey (Su et al., 1998) conducted in the year 1993, 8 years earlier than our study, revealed the adjusted prevalence rate of active epilepsy was 2.64/1000 above 40 years old. Higher prevalence rate was noted in males in both studies. The leading cause for remote symptomatic cases in both studies was head injury. Moreover, the age-specific prevalence rates are rather similar in Keelung and Ilan. Although using different methods of investigation, it seems that the patterns of epilepsy in adults are quite similar in these two populations in Taiwan. These findings may suggest that there is no substantial change of the magnitude and

Table 3

Age-specific etiologies of active epilepsy in Keelung city, Taiwan

\begin{tabular}{lllllllr}
\hline Age & Head injury & Stroke & CNS infection & Prenatal injury & Brain tumor & Idiopathic/cryptogenic & Total \\
\hline $30-39$ & 2 & 0 & 0 & 1 & 0 & 6 & 9 \\
$40-49$ & 2 & 0 & 2 & 1 & 1 & 8 & 14 \\
$50-59$ & 0 & 0 & 1 & 0 & 0 & 2 & 3 \\
$60-69$ & 0 & 1 & 0 & 0 & 0 & 4 & 5 \\
$70-79$ & 1 & 1 & 0 & 0 & 0 & 3 & 5 \\
$80^{+}$ & 0 & 0 & 0 & $2(5.4 \%)$ & $1(2.7 \%)$ & $24(64.9 \%)$ & 1 \\
Total & $5(13.5 \%)$ & $2(5.4 \%)$ & $3(8.1 \%)$ & & & $37(100 \%)$ \\
\hline
\end{tabular}


causes of this disorder in adults over the past decade in northern Taiwan. Few studies addressed the change of prevalence rates over time. In a longitudinal study in the UK, Cockerell et al. (1995) reported similar prevalence rates of lifetime epilepsy in 1983 and 1993. However, an increased prevalence rate over time was noted and can be attributed to improved case identification in the latest time periods in Minnesota (Hauser et al., 1991).

\subsection{Etiologies of active epilepsy}

Head injury is the leading cause of symptomatic cases in our study and in Ilan, as found in previous reports (Haerer et al., 1986; Li et al., 1985; Oun et al., 2003). The majority of our patients with head injury are male and young. Head injuries are often caused by accidents. In Taiwan, traffic accident was the major cause of head injury (Chiu et al., 1997), and motorcyclists accounted for the vast majority of head injury cases among traffic accident victims (64.5\%). It is well known that head injury is a major and preventable cause of epilepsy (Annegers et al., 1998; Frey, 2003). Our study reflected the importance of improving public traffic safety reduce head injuries and thus avoid potential complications like epilepsy.

\subsection{Age-specific prevalence rates}

Fig. 2 shows the age-specific prevalence rates in different studies. In this survey, the prevalence rate was highest in age group 40-50 years old and not elevated at advanced age. This pattern was similar to that in China (Li et al., 1985), Ilan (Su et al., 1998) and Bombay, India (Bharucha et al., 1988). In some reports, there was a steady increase in prevalence rates with age observed in Minnesota, USA (1980) (Hauser et al., 1991) and Nigeria (Osuntokun et al., 1987). However, this was not seen in our study and some other studies (Keranen et al., 1989; Maremmani et al., 1991; Forsgren, 1992; Oun et al., 2003). As mentioned before, the high incidence rate in the young group is due to head injury. And the lower prevalence rate in old age compared to western countries may be also attributed to underlying etiology related to stroke, which was the most common cause for epilepsy reported in the elderly (Luhdorf et al., 1986) and the incidence rate in our country is lower than that in western countries.

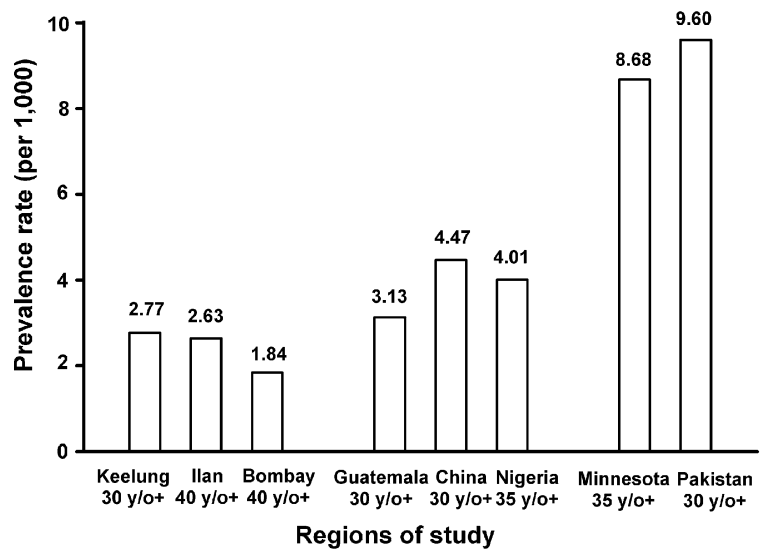

Fig. 3. Age-adjusted prevalence rates of adult patients with active epilepsy between different studies. To compare results between studies, we recalculated the prevalence rates using similar range of age. The prevalence rates were all adjusted to US 1980 population shown above the top of bar.

\subsection{Comparison of prevalence rates with previous studies}

Fig. 3 shows the calculated prevalence rate of adult patients with active epilepsy in different studies with similar range of age. Higher prevalence rates were noted in Pakistan and Minnesota, USA (Hauser et al., 1991). Median prevalence rate was noted in Nigeria (Osuntokun et al., 1987), China (Li et al., 1985) and Guatemala (Mendizabal and Salguero, 1996). Our data (2.77/1000), together with that in Ilan (Su et al., 1998) and in Bombay (Bharucha et al., 1988) were lower than other reports.

Two possibilities account for a lower prevalence rate found in our study. Firstly, different definitions may render direct comparison unavailable. Some studies only included active epilepsy whereas some covered seizure as well as epilepsy. Secondly, unidentified cases and concealment due to social stigma may lead to underreported cases (Osuntokun et al., 1987; Hauser et al., 1991; Aziz et al., 1994). In our study, the former is unlikely because our study was a community-based survey with one-stage method. However, concealment is still probable because there is more psychosocial pressure toward patients with epilepsy in Chinese culture regions than in western countries (Caveness and Gallup, 1980; Lai et al., 1990; Jensen and Dam, 1992; Chung et al., 1995; Liou et al., 2005). Lower rate of 
epilepsy may be due to the fact that participants undergoing neurological examination may deny or underreport symptoms and signs related to epilepsy because of stigma.

In our study, up to $24.32 \%(9 / 37)$ adult patients of active epilepsy were first detected in this survey. Because they were unaware of the meaning or importance of the clinical symptoms, they did not visit physicians to receive any traditional or modern therapy against epilepsy. This may affect their working performance and quality of life. In a recent report from China, up to $41 \%$ of patients with epilepsy do not receive medical treatment (Wang et al., 2003). These facts highlight the importance of finding hidden patients and giving adequate treatment.

\section{Conclusion}

In conclusion, the prevalence rate of epilepsy was rather stable over the past decade in Taiwan. The common pattern of adult patients epilepsy mainly attributed to head injury across time also underscores the importance of improving public safety in the prevention of epilepsy cases.

\section{Acknowledgments}

We are indebted for those who were involved in this work, including Yao-Der Chen (Director, Health Bureau of Keelung City), Ting-Ting Wang (Chief of Health Prevention Division, Health Bureau of Keelung City) and Health Informatics Group in Health Bureau of Keelung City. This work is supported by National Science Council NSC-92-2320-B-002-070 and 932320-B-002-028, Taipei, Taiwan.

\section{Appendix A}

The questions in the screening questionnaire are:

1. Have you ever experienced or been found unconsciousness before?

2. (If yes in Q1) At that time, did anybody notice that you fell suddenly with shouting initially, and had rigidity and/or convulsions of your limbs or face?
3. Have anybody told you that you had eyes starring, impaired response, or stopping ongoing movement/work in a brief period (seconds to minutes)?

4. Have you ever heard of seizure or epilepsy?

5. Have you ever been diagnosed as a patient of seizure or epilepsy?
(If yes) (1) When?
(2) In which hospital?
(3) Have you taken drugs?
(4) How long is the seizure-free period?

\section{References}

Annegers, J.F., Hauser, W.A., Coan, S.P., Rocca, W.A., 1998. A population-based study of seizures after traumatic brain injuries. N. Engl. J. Med. 338, 20-24.

Aziz, H., Ali, S.M., Frances, P., Khan, M.I., Hasan, K.Z., 1994. Epilepsy in Pakistan: a population-based epidemiologic study. Epilepsia 35, 950-958.

Bharucha, N.E., Bharucha, E.P., Bharucha, A.E., Bhise, A.V., Schoenberg, B.S., 1988. Prevalence of epilepsy in the Parsi community of Bombay. Epilepsia 29, 111-115.

Caveness, W.F., Gallup Jr., G.H., 1980. A survey of public attitudes toward epilepsy in 1979 with an indication of trends over the past thirty years. Epilepsia 21, 509-518.

Chen, T.H., Chiu, Y.H., Luh, D.L., Yen, M.F., Wu, H.M., Chen, L.S., Tung, T.H., Huang, C.C., Chan, C.C., Shiu, M.N., Yeh, Y.P., Liou, H.H., Liao, C.S., Lai, H.C., Chiang, C.P., Peng, H.L., Tseng, C.D., Yen, M.S., Hsu, W.C., Chen, C.H., 2004. Communitybased multiple screening model: design, implementation, and analysis of 42,387 participants. Cancer 100, 1734-1743.

Chiu, W.T., Yeh, K.H., Li, Y.C., Gan, Y.H., Chen, H.Y., Hung, C.C., 1997. Traumatic brain injury registry in Taiwan. Neurol. Res. 19, 261-264.

Chiu, Y.H., Chen, L.S., Chan, C.C., Liou, D.M., Wu, S.C., Kuo, H.S., Chang, H.J., Chen, T.H., 2006. Health information system for community-based multiple screening in Keelung, Taiwan (Keelung community-based integrated screening no. 3). Int. J. Med. Inform. 75, 369-383.

Chung, M.Y., Chang, Y.C., Lai, Y.H., Lai, C.W., 1995. Survey of public awareness, understanding, and attitudes toward epilepsy in Taiwan. Epilepsia 36, 488-493.

Cockerell, O.C., Eckle, I., Goodridge, D.M., Sander, J.W., Shorvon, S.D., 1995. Epilepsy in a population of 6000 re-examined: secular trends in first attendance rates, prevalence, and prognosis. $\mathrm{J}$. Neurol. Neurosurg. Psychiatry 58, 570-576.

Forsgren, L., 1992. Prevalence of epilepsy in adults in northern Sweden. Epilepsia 33, 450-458.

Forsgren, L., Beghi, E., Oun, A., Sillanpaa, M., 2005. The epidemiology of epilepsy in Europe-a systematic review. Eur. J. Neurol. 12, 245-253.

Frey, L.C., 2003. Epidemiology of posttraumatic epilepsy: a critical review. Epilepsia 44 (Suppl. 10), 11-17.

Gracia, F., de Lao, S.L., Castillo, L., Larreategui, M., Archbold, C., Brenes, M.M., Reeves, W.C., 1990. Epidemiology of epilepsy 
in Guaymi Indians from Bocas del Toro Province, Republic of Panama. Epilepsia 31, 718-723.

Haerer, A.F., Anderson, D.W., Schoenberg, B.S., 1986. Prevalence and clinical features of epilepsy in a biracial United States population. Epilepsia 27, 66-75.

Hauser, W.A., Annegers, J.F., Kurland, L.T., 1991. Prevalence of epilepsy in Rochester, Minnesota: 1940-1980. Epilepsia 32, 429-445.

Hsu, W.C., Chiu, Y.H., Chiu, H.C., Liou, H.H., Jeng, Y.C., Chen, T.H., 2005. Two-stage community-based screening model for estimating prevalence of diabetic polyneuropathy (KCIS no. 6). Neuroepidemiology 25, 1-7.

ILAE, 1981. Proposal for revised clinical and electroencephalographic classification of epileptic seizures. From the Commission on Classification and Terminology of the International League Against Epilepsy. Epilepsia 22, 489501.

ILAE, 1993. Guidelines for epidemiologic studies on epilepsy. Commission on Epidemiology and Prognosis, International League Against Epilepsy. Epilepsia 34, 592-596.

ILAE, 1997. ILAE Commission Report. The epidemiology of the epilepsies: future directions. International League Against Epilepsy. Epilepsia 38, 614-618.

Jensen, R., Dam, M., 1992. Public attitudes toward epilepsy in Denmark. Epilepsia 33, 459-463.

Keranen, T., Riekkinen, P.J., Sillanpaa, M., 1989. Incidence and prevalence of epilepsy in adults in eastern Finland. Epilepsia 30, 413-421.

Lai, C.W., Huang, X.S., Lai, Y.H., Zhang, Z.Q., Liu, G.J., Yang, M.Z., 1990. Survey of public awareness, understanding, and attitudes toward epilepsy in Henan province, China. Epilepsia 31, 182-187.
Li, S.C., Schoenberg, B.S., Wang, C.C., Cheng, X.M., Zhou, S.S., Bolis, C.L., 1985. Epidemiology of epilepsy in urban areas of the People's Republic of China. Epilepsia 26, 391-394.

Liou, H.H., Chen, R.C., Chen, C.C., Chiu, M.J., Chang, Y.Y., Wang, J.D., 2005. Health related quality of life in adult patients with epilepsy compared with a general reference population in Taiwan. Epilepsy Res. 64, 151-159.

Luhdorf, K., Jensen, L.K., Plesner, A.M., 1986. Etiology of seizures in the elderly. Epilepsia 27, 458-463.

Maremmani, C., Rossi, G., Bonuccelli, U., Murri, L., 1991. Descriptive epidemiologic study of epilepsy syndromes in a district of northwest Tuscany, Italy. Epilepsia 32, 294-298.

Mendizabal, J.E., Salguero, L.F., 1996. Prevalence of epilepsy in a rural community of Guatemala. Epilepsia 37, 373-376.

Osuntokun, B.O., Adeuja, A.O., Nottidge, V.A., Bademosi, O., Olumide, A., Ige, O., Yaria, F., Bolis, C.L., Schoenberg, B.S., 1987. Prevalence of the epilepsies in Nigerian Africans: a communitybased study. Epilepsia 28, 272-279.

Oun, A., Haldre, S., Magi, M., 2003. Prevalence of adult epilepsy in Estonia. Epilepsy Res. 52, 233-242.

Su, C.L., Chang, S.F., Chen, Z.Y., Lee, C.S., Chen, R.C., 1998. Neuroepidemiological survey in Ilan, Taiwan (NESIT): (4) prevalence of epilepsy. Acta Neurol. Taiwanica 7, 75-84.

Tung, T.H., Chiu, Y.H., Chen, L.S., Wu, H.M., Boucher, B.J., Chen, T.H., 2004. A population-based study of the association between areca nut chewing and type 2 diabetes mellitus in men (Keelung community-based integrated screening programme no. 2). Diabetologia 47, 1776-1781.

Wang, W.Z., Wu, J.Z., Wang, D.S., Dai, X.Y., Yang, B., Wang, T.P., Yuan, C.L., Scott, R.A., Prilipko, L.L., de Boer, H.M., Sander, J.W., 2003. The prevalence and treatment gap in epilepsy in China: an ILAE/IBE/WHO study. Neurology 60, 1544-1545. 